\title{
PAEDIATRICS
}

\section{Kidney disease in youth-onset type 2 diabetes mellitus}

Risk of kidney failure is fourfold greater in individuals with youth-onset type 2 diabetes mellitus (T2DM) than in those with type 1 diabetes mellitus (T1DM), report researchers from Canada.

Prevalence of youth-onset T2DM is increasing. However, the rates and time to progression to kidney disease in these young individuals compared with those in their peers with T1DM or without diabetes mellitus are not well studied.

The researchers used health-care records to evaluate long-term renal complications and renal survival in patients with T2DM $(n=342)$ or T1DM $(n=1,011)$. These cohorts included children and adolescents aged $1-18$ years who were seen at a diabetes educational resource centre between 1986 and 2007. In addition, 1,710 control individuals without diabetes mellitus were matched by age, sex and postal code to the T2DM cohort.
Patients with youth-onset T2DM had a fourfold increased risk of renal failure compared with those with T1DM, after adjustment for age at diagnosis, $\mathrm{HbA}_{1 \mathrm{c}}$, BMI z score and era of diagnosis. Albuminuria and renin-angiotensinaldosterone-system inhibitors were risk factors associated with renal failure. Compared with control individuals, patients with youth-onset T2DM had a 23-fold and 39-fold higher risk of renal failure and dialysis, respectively. Renal survival at 15 years was $100 \%$ in the T1DM group but only $92 \%$ in the T2DM group.

The high burden of kidney disease in youth-onset T2DM highlights a critical need for further research.

Carol Wilson

Original article Dart,A. B. et al. High burden of kidney disease in youth-onset type 2 diabetes. Diabetes Care doi:10.2337/dc11-2312 